\title{
INVESTIGACIÓN
}

Recibido: 12/03/2021 --- Aceptado: 15/04/2019 --- Publicado: 24/05/2021

\section{ESTRUCTURALISMO DE PROPP EN LOS PERIODISTAS HÉROES Y VILLANOS DEL CINE ESPAÑOL}

\section{Propp's structuralism in the journalists heroes and villains in the spanish cinema}

Cristina San José de la Rosa1: Universidad de Valladolid. España. cristina.sanjose@uva.es.

\section{Cómo citar el artículo:}

San José de la Rosa, C. (2021). Estructuralismo de Propp en los periodistas héroes y villanos del cine español. Vivat Academia. Revista de Comunicación, 154, 245-262. http://doi.org/10.15178/va.2021.154.e1350

\section{RESUMEN}

Los personajes periodistas héroes y villanos ocupan un lugar destacado en el cine español y su estudio aporta una visión de la profesión desde la perspectiva que brinda el rico caleidoscopio del cine. Esta investigación estudia 135 películas en siete décadas, desde 1942 hasta 2012, lo que proporciona una inusual panorámica de la evolución del informador y de los medios de comunicación a lo largo del último siglo en nuestro país. La originalidad de la metodología se encuentra en la recuperación de Morfología del cuento (1928) de Vladimir Propp para trasladar el estructuralismo de la literatura a la narrativa audiovisual, una semiosis que configura una exclusiva morfología del periodista. El autor ruso Propp encontró 31 funciones matrices que se repiten en los cuentos del folclore de su país y este trabajo 21 funciones en las películas. El detallado análisis de guiones, secuencias y encuadres se sintetiza en esferas y acciones en las que se mueven los informadores. Héroes y villanos que conforman un novedoso reflejo en la pantalla del periodismo español con un dominio del medio de comunicación tradicional de la prensa escrita, del personaje masculino y del periodista envuelto en una vida de caos emocional y adicciones.

\footnotetext{
${ }^{1}$ Cristina San José de la Rosa: Doctora profesora en el Grado de Periodismo y en el Máster de Cine de la Universidad de Valladolid. Licenciada en Ciencias de la Información (Salamanca) y en Filología Hispánica (Salamanca). Trayectoria profesional durante 22 años en varios medios de comunicación. Tesis doctoral sobre periodismo en el cine español.
} 


\section{PALABRAS CLAVE}

Rol del periodista - Periodismo y Cine - Cine español - Estructuralismo - Vladimir Propp - Narrativa audiovisual - Héroes y villanos en el cine - Estereotipos sexistas Adicciones.

\section{ABSTRACT}

Hero and villain journalist characters occupy a prominent place in Spanish cinema and their study provides a vision of the profession from the perspective provided by the rich kaleidoscope of cinema. This research studies 135 films in seven decades, from 1942 to 2012, which provides an unusual overview of the evolution of the news reporter and the media over the last century in our country. The originality of the methodology is found in the recovery of Morphology of the story (1928) by Vladimir Propp to transfer the structuralism of literature to audiovisual narrative, a semiosis that configures an exclusive morphology of the journalist. The Russian author Propp found 31 matrix functions that are repeated in the folk tales of his country and this work 21 functions in the movies. The detailed analysis of scripts, sequences and frames is synthesized in spheres and actions in which the informants move. Heroes and villains that make up a new reflection on the screen of Spanish journalism with a mastery of the traditional means of communication of the written press, the male character and the journalist involved in a life of emotional chaos and addictions.

\section{KEYWORDS}

Journalist's role - Journalism and Cinema - Spanish cinema - Structuralism - Vladimir Propp - Audiovisual narrative - Heroes and villains in the cinema - Sexist stereotypes - Addictions.

\section{INTRODUCCIÓN}

El escenario ilimitado del cine abre la posibilidad de un estudio de la evolución del periodismo español a través de las películas, material clave que convierte la cinematografía del país en espacio para un fructífero y particular análisis. Los relatos fílmicos de los héroes y villanos de la prensa ofrecen otra visión a la historia de la profesión muy necesaria para la investigación. Este trabajo aporta la originalidad de rescatar al teórico del estructuralismo Vladimir Propp y trasladar a la narrativa audiovisual un método clásico de análisis literario, una semiosis que configura una exclusiva morfología del periodista.

El autor ruso propuso en su obra Morfología del cuento (1928) que existen 31 funciones irreductibles que se repiten en los cuentos de su país. Consideró que, si la morfología botánica configura las formas de las plantas, su libro detalla las formas de los cuentos tradicionales (1971). Ahora este trabajo, inspirado en el teórico estructuralista, sienta las bases formales del periodista en el cine español y muestra cómo las películas pueden aunar tradición literaria con nuevas tecnologías y se convierten en documento fundamental en el ámbito universitario, con conocimientos 
para el periodismo y la cinematografía, ideas constatadas ya por autores como Merchán o Evans.

La presente investigación se centra en 135 películas españolas con informadores de prensa, radio y televisión como personajes principales o secundarios desde 1942 hasta 2012. Se trata de 7 décadas de cine en las que se presta especial atención a su presencia como héroes o villanos, dos categorías recurrentes en el cine universal que se intentará demostrar que también aparecen en la cinematografía española con periodistas.

\subsection{Héroes y villanos en el cine}

Los autores anglosajones plantean una clara dicotomía héroes o villanos que se plasma ya en los años setenta en Stop the presses! The newspaperman in American Films, obra de Alex Barris de 1976. Es un referente y prueba de ello es que Journalists in filme. Heroes and villains, de Brian McNair, coincide en algunos perfiles. Como su título indica, defiende la misma diferencia que se plantea en nuestro trabajo de investigación: buenos y malos en la información. Entre los héroes encuentra cuatro: "perros guardianes", testigos en primera línea de los hechos, heroínas y artistas. Entre los villanos destaca tres: granujas, estafadores y fabricantes de reyes. Halla 2.166 en EEUU desde el cine mudo hasta el año 1996 (2010).

¿Héroes o villanos? Parece que la respuesta es clara tras comprobar que tan solo el $20 \%$ de las películas cuentan con villanos mientras que en el resto el periodista es un claro héroe social. Pero McNair quiere dejar patente que los límites no resultan tan precisos y recuerda que en ocasiones un villano comienza siendo héroe o, en otro extremo, un bondadoso periodista termina como el más malvado ser humano. Recuerda que ocurre en un histórico como Citizen Kane (Ciudadano Kane, Orson Welles, 1941), película en la que Charles Foster Kane pasa por cuatro estados: víctima, idealista, cínico y monstruo (2010).

El profesor de la Universidad de Illinois (Chicago) Matthew C. Ehrlich de nuevo ofrece la dicotomía héroes y villanos en Journalism in the movies. Considera que la labor de la prensa en la sociedad americana tiene que ser la de destapar mentiras y servir a la democracia y así lo refleja el cine, aunque esta tarea se degrada cuando el periodista elige el camino incorrecto y se convierte en villano (2006).

Profesor de Periodismo en la Universidad de Nueva York, Howard Good es el autor más prolífico en la relación cine y periodista y arroja títulos de especial interés como son Outcasts: The image of journalists in contemporaryfilme en 1989 y Journalism Ethics goes to the movies en 2008. Detecta periodistas como víctima, como comediante, como penitente o como caballero.

Existen otras publicaciones con héroes y villanos como las realizadas por Lucía Tello pero ya sin la clara diferenciación héroes y villanos, aunque siempre hay esa tendencia a distinguir buenos y malos. De nuevo Ehrlich realiza una revisión al tema

Vivat Academia. Revista de Comunicación. 2021, n 154, 245-262 
en 2015 con Heroes and Scoundrels: The Image of the Journalist in Popular Culture, libro que firma con Joe Saltzman. Consideran que los periodistas de la cultura popular han creado la actual visión de la prensa y su papel en una sociedad libre. Centran su análisis en retratos de periodistas en televisión, cine, radio, novelas, cómics, obras de teatro y otros medios de comunicación. San José-De la Rosa, Miguel-Borrás y GilTorres también estudian la presencia del periodista en el cine (2019 y 2020).

\subsection{La construcción narrativa del cine}

Como el resto de las artes, el cine encierra la expresión de un contenido interno, es un sistema articulado de signos inmerso en una estructura determinada. El cine es una instancia narrativa, una manifestación de un discurso no marcado en el que existe una interpelación de los signos icónicos, gestuales y verbales y sus articulaciones a través del montaje, el movimiento interno o el movimiento de cámara para conseguir su "grandeza” (Miguel Borrás, 2008: 4).

Introducción al análisis estructural del relato (2011), texto fundacional en el estudio y análisis de los procesos narrativos escrito en 1970 por Roland Barthes, recoge que, ese conjunto de frases que conforman el relato puede tener como soporte el lenguaje articulado, ya sea oral o escrito, pero también una imagen, fija o móvil, o un gesto, o todos ellos combinados, lo que da lugar a novelas, cuadros, noticias y también películas.

Según defienden Aumont, Bergara, Marie y Vernet la "excelente relación" entre cine y narración no fue tan clara al principio puesto que no se consideró el cine "masivamente narrativo" sino que podría haber sido un instrumento de investigación científica, un útil del reportaje o el documental, una prolongación de la pintura o una distracción sin más. Los autores destacan tres razones que hacen que cine y narración confluyan: la imagen móvil figurativa, la imagen en movimiento y la búsqueda de una legitimidad (1985: 89-91).

La gran pantalla es una máquina de contar historias, es el gran medio de comunicación de nuestro siglo y por tanto es un eficiente y muy utilizado dispositivo narrativo. Mirar la imagen cinematográfica equivale a mirar el devenir, el paso del tiempo y el movimiento. Señala José Enrique Monterde que en La imagen tiempo (1985), texto fundamental del filósofo Gilles Deleuze, la acción es el elemento transformador de una situación, por lo que el cine entra de lleno en el universo de la narración. Recupera también a Todorov, que indica que la narración consiste en pasar de una relación de sucesión entre unidades a otra relación de transformación al cambiar una situación inicial a una nueva (2001: 20). Orellana habla del drama humano en el cine contemporáneo y plantea que es una "forma de narrar al hombre, de contar con historias cómo está hecho el ser humano". A través de la acción de los personajes se deduce su naturaleza, sus deseos y preocupaciones. Mientras que la literatura requiere una ardua elaboración gracias a una pluma maestra, el cine es un reflejo especular de unos hechos (2004: 17-18). 
Una máquina de plasmar historias sometida a continuos cambios a los largo de su existencia y desde hace unos años adaptándose a la realidad virtual, como anticipa la profesora Mercedes Miguel Borrás en su revisión del Séptimo Arte: "El discurso sobre el realismo necesita una renovación para poder legitimarse" (2013: 34). Sostiene la posibilidad de una vuelta a los orígenes en la concepción fílmica para recuperar la esencia del cine y así se entiende el éxito en los últimos años de The Artist (Michel Hazanavicius, 2011) y Blancanieves (Pablo Berger, 2012), películas mudas que parece que se apuntan a la postura de Chaplin cuando se negó a utilizar el sonido incluso diez años después de su aparición porque "despojaba al cine de su fuerza expresiva" (2014: 35).

\subsection{Analizar las películas como relatos}

El relato está presente tanto en el lenguaje oral como en el lenguaje escrito, tanto en leyendas y cuentos relatados oralmente que se transmiten generación tras generación, como en novelas, en cómics y por supuesto también en la disciplina que ocupa esta investigación, el cine. Ante el amplio repertorio de relatos que brinda el infinito universo de las palabras, la forma de abordar su estudio puede ser muy diversa: desde el punto de vista histórico, lingüístico, psicológico, estético o filosófico.

Para abordar este tema, esencial en el devenir de nuestra investigación, se toma como referente Morfología del cuento, publicado por Vladimir Propp en 1928, texto fundacional en el estudio de los relatos, retomado en los años 60 por Barthes y Greimas, entre otros. Ha sido ampliamente estudiado para poder efectuar su transposición al lenguaje cinematográfico por Aumont y Marie en Análisis del filme (1988). El argumento que presenta una película es un pretexto para las conversaciones de la población, para la crítica periodística e incluso para la formación de futuras generaciones. Así lo pretende demostrar la presente investigación de utilidad para la enseñanza y el aprendizaje en las facultades de Periodismo. Recuerdan los teóricos que la educación también utiliza el cine para crear mensajes y es frecuente encontrar en las aulas la proyección de Las uvas de la ira (The Grapes of Wrath, John Ford, 1940) para hablar de la crisis de los años 30, o de La gran ilusión (La Grande Illusion, Jean Renoir, 1937) para la I Guerra Mundial (1988: 131).

Aumont y Marie sostienen que es necesario estudiar el análisis estructural para remediar el "exceso de arbitrariedad" que implica el análisis temático (1988: 135). Proponen varios modelos creados para el análisis de obras literarias que permiten su transposición al terreno de la filmografía, una oferta que comienza con Propp, un autor clave de referencia obligada para nuestra investigación.

Con su Morfología del cuento, fue el primer autor que plantea un doble principio analítico y estructural a la hora de estudiar el conjunto de los cuentos maravillosos del folclore ruso. Descompone cada cuento en unidades abstractas para establecer después las posibles combinaciones y realizar una clasificación de esas 
combinaciones. Llamó a cada unidad básica 'función', que es cada momento elemental del relato que corresponde a una única acción simple y que puede encontrarse en gran número de cuentos. El autor ruso estableció 31 funciones que a su vez agrupa en siete esferas de acción o 'roles': el agresor, el donante o proveedor, el auxiliar, la princesa o personaje al que se busca, el mandatario, el héroe y el falso héroe. Afirma que el cuento maravilloso es una sucesión de secuencias de funciones que parten de un daño o de una falta y obedecen a una serie de obligaciones que limitan el número de relatos posibles (Propp, 2011).

\subsection{El periodista como personaje recurrente}

Lucía Tello recuerda que desde que el cinematógrafo se instaló en el país a finales del siglo XIX hasta 1913 no aparecen periodistas. Sin embargo, entre ese año y 1939 hay más de una decena, "cifras nada desdeñables teniendo en cuenta la escasa productividad de nuestra industria en sus tiempos inaugurales" (2016: 15).

La figura del periodista resulta atractiva para los cineastas dado que en el informador se aúnan varias características: es capaz de vivir experiencias inaccesibles para el resto de la sociedad con su presencia en acontecimientos importantes o entrevistas a personas interesantes para después escribir o contarlo; ejerce como contrapoder un contrapoder, tienen una autoridad que permite vigilar (y enfrentarse cuando es necesario) a los poderosos y está a disposición de las exigencias de la profesión con sus noticias imprevistas y horarios imposibles que suelen tener consecuencias en su vida personal. Esto llevó al corresponsal Manuel Leguineche a hablar de los reporteros de guerra como la tribu de las tres "des": dipsómanos, divorciados y deprimidos (Mera: 2008).

Es más, un estudio realizado por Ángeles Pastor sobre las motivaciones de los alumnos de Periodismo a la hora de elegir su carrera profesional da la clave sobre el interés social de la profesión entre los jóvenes y su estrecha relación con el cine puesto que concluye que los adolescentes tienen las referencias de la profesión que absorben de las televisión o el cine: "La personalidad profesional del periodista es una de las más tipificadas a ojos de los jóvenes, algo así como pudieran ser la del maestro o la del médico (Pastor, 2010: 200).

\section{OBJETIVOS E HIPÓTESIS}

La investigación se plantea los siguientes objetivos:

1) Identificar el perfil del periodista que presentan las películas españolas a través de la creación de una morfología con las funciones de los informadores como personajes principales y secundarios durante siete décadas, de 1942 a 2012.

2) Delimitar un retrato del periodista en su profesión con las características que permiten distinguir entre héroes y villanos y el género cinematográfico en el que se enmarcan. 
3) Diferenciar los tipos de héroes y villanos que presentan las películas españolas a lo largo del tiempo para delimitar su evolución desde la posguerra hasta la actualidad.

4) Observar con detalle la diferencia en el tratamiento de los héroes y villanos según sean hombres o mujeres.

Con estos objetivos, la investigación se plantea los siguientes objetivos:

H1. Las funciones del periodista en las películas españoles reflejan la dicotomía entre héroes enmarcados en el género drama y villanos en el género comedia

H2. La prensa escrita es el medio de comunicación dominante en las películas españolas con profesionales de la información

H3. La representación de los periodistas en el cine español perjudica a las mujeres y favorece a los hombres.

\section{METODOLOGÍA}

Una de las aportaciones originales de esta investigación, como ya se ha insistido a lo largo del trabajo, es la recuperación del libro Morfología del cuento (Morfológuiya skazki), reconocida obra del ruso Vladimir Propp (1895-1975) publicada en 1928, como base para crear una morfología del periodista. Su estudio de los 100 cuentos maravillosos del folclore de su país establece la matriz originaria de la que surgen el resto de relatos universales, con un conjunto de elementos narrativos irreductibles que denominó 'funciones de los personajes', 31 en total. En nuestro estudio, tras el análisis de las 135 películas españolas con periodistas se obtiene una morfología del comunicador con 21 funciones (San José, 2017).

En la primera fase del estudio se emplean métodos cuantitativos con la definición y acotación de la muestra. El trabajo se sustenta en una amplia recopilación de largometrajes de producción española con periodistas. Una intensa búsqueda y localización desde los inicios del cine español hasta la actualidad sirvió para centrar la filmografía 'general' con los 220 títulos. Después se elaboró una filmografía 'específica' con la selección de los títulos con papeles de principal o secundario hasta ajustar la cifra en 135, una importante información para esta investigación que se incluye en el apartado de material complementario con la tabla de las películas analizadas (TABLA 1 MATERIAL COMPLEMENTARIO).

La escasez de bibliografía sobre periodismo en el cine español hizo que la base de datos de la Filmoteca Española se convierte en el instrumento más fiable para localizar las películas, a través de la búsqueda con las palabras clave informador, periodista, radio, televisión, cámara, fotógrafo, prensa, periódico y periodismo.

El visionado de todo el cine posible en español, tanto si se tienen referencias de periodismo en su contenido como si se carece de ellas, es el método más fiable para encontrar a los periodistas, un trabajo que se ha realizado durante años y sigue abierto al finalizar esta investigación. 
Desde las primeras películas se recogió la información en fichas de análisis en dos bases de datos a través del programa informático Filemaker:

- Base de datos 1: Fichas de análisis fílmicas: base de datos general con la información sobre la película. Los campos y formulario utilizados reproducen los que plantea la base de datos de la Filmoteca Española.

- Base de datos 2: Fichas de análisis del periodista: base de datos específica de periodismo con datos fundamentales para la esta investigación. Los campos y formulario incluyen aspectos relacionados con el papel del periodista además de información de la base de datos 1.

Comienza la muestra en 1942 puesto que ese año se localizan varias películas con periodista en España. Se cierra en 2012 para abarcar así un tiempo redondeado de siete décadas. Se optó por cerrar el número de 135 puesto que se observó que las funciones para crear la morfología se repetían, momento en el que se consideró que ya se habían extraídos suficientes 'funciones irreductibles' en los personajes, como le ocurrió a Propp con los cuentos de la tradición rusa.

Las 21 funciones se distribuyen en tres esferas.

Primera esfera: según la sección en la que desempeña su profesión.

Segunda esfera: según la personalidad del periodista.

Tercera esfera: según se consideren héroes o villanos.

La presentación de los datos se realiza con las siguientes pautas:

- La definición de la acción lo más precisa posible en las 21 funciones.

- El signo convencional con una letra en mayúscula que indica la función.

- El número para el medio de comunicación: prensa (1), radio (2) y televisión (3).

- Las indicaciones Pr y Sec para principales y secundarios respectivamente.

Las películas se ordenan de forma cronológica según los periodos marcados en esta investigación: Posguerra POS (desde el año 1942 hasta 1959), Desarrollismo DES (desde 1960 hasta 1974), Transición y los 80 TRA (desde 1975 hasta 1989), Modernidad MOD (desde 1990 hasta 1999), Últimos años UA (desde 2000 a 2012). De este modo y en consecuencia con los objetivos, se irá verificando la evolución.

En el apartado de material complementario de esta investigación se incluye la tabla con las funciones de la morfología del periodista en el cine español (TABLA 2 MATERIAL COMPLEMENTARIO). Aunque la combinación de definiciones y letras supone un complejo entramado con multitud de lecturas e información sobre periodismo y cine, para esta investigación debe acotarte sobre todo a las definiciones de la segunda esfera (género cinematográfico) y de la tercera (héroes y villanos). 
La primera esfera es 'Sección en el medio de comunicación' e incluye 9 definiciones: entretenimiento, sucesos, investigación y política, compromiso, guerra, biografía, fenómenos paranormales, director o jefe y becario.

La segunda esfera, 'Personalidad', ofrece 7 definiciones y son más complejas: desequilibrio emocional masculino, drama, thriller, terror; desequilibrio emocional femenino, drama, thriller, terror; desequilibrio emocional masculino, comedia; desequilibrio emocional femenino, comedia; estrés laboral; amor y desamor.

Por último, la esfera tercera, 'Héroes y villanos', acoge 5 definiciones, también complejas: héroe, bondad, bien; héroe, verdad, peligro; héroe, inverosimilitud; villano, sin ética, sensacionalismo, maldad, mal y, finalmente, villano, inverosimilitud, sátira.

\section{RESULTADOS}

Tras realizar un exhaustivo rastreo en la bibliografía sobre el tema, con la información de las fichas de análisis presentadas en la metodología y las funciones según la morfología del periodista en el cine español según Propp, se procede al estudio de las 135 películas y se detecta que en 98 existen personajes con suficiente identidad como para formar parte de la división establecida entre héroes y villanos. Con el propósito de poder elaborar con criterio esta clasificación, se establece una separación entre periodistas en situaciones realistas -que pueden aparecen en cualquier redacción de un medio de comunicación- y periodistas en situaciones no realistas -personajes inverosímiles por presentar situaciones extremas, de ciencia ficción o exceso de sátira-. Las películas aparecen con esta división en tablas en material complementario de la investigación (TABLA 3 MATERIAL COMPLEMENTARIO).

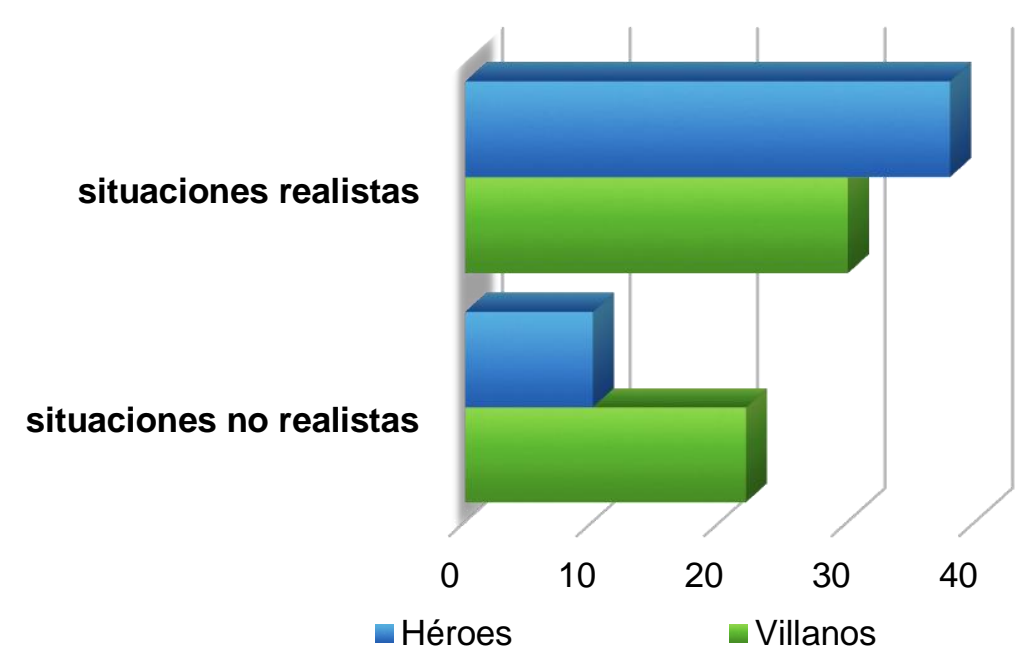

Gráfico 1. Héroes y villanos 


\subsection{Periodista héroe en situaciones realistas}

Los periodistas héroes que presenta el cine español a lo largo de los 70 años analizados se adaptan a los nuevos tiempos. De las 66 películas en las que aparecen en situaciones realistas en las redacciones de prensa, radio y televisión, en 38 casos son héroes.

El drama domina en las historias de los años 40 y 50 con los primeros informadores héroes y es el varón quien lleva el peso en las redacciones de los periódicos, aún sin rastro de radio y televisión en este periodo. Se muestran mujeres con papel secundario (Escuela de Periodismo) y se detecta la intención de colocar a las periodistas al lado de la información frívola puesto que ellas son las encargadas de realizar preguntas relacionadas con la vida personal del entrevistado.

En los años 60, el tono dramático continúa para las historias con héroes aunque por primera vez aparecen el thriller y la acción para resaltar la heroicidad y valentía del informador. Los hombres protagonizan las escenas y de nuevo el papel de una mujer, en este caso en La Redada, sirve para mostrar el lado más frívolo de la información. La prensa escrita es la que manda, sin radio y televisión aún en las películas analizadas.

El año 1975 es un momento de grandes cambios en la historia del país y también en el cine con periodistas. Es cuando en el apartado de héroes surge con fuerza el periodismo ideológico y también con ímpetu aparece un medio de comunicación, la radio. La Transición muestra drama en sus 9 películas con héroes aunque en tres ocasiones combinado con thriller y surge el primer caso de comedia dramática en Corazón de papel. Precisamente es en esta película y en otra de esta etapa, La verdad sobre el caso Savolta, en las que el periodista héroe llega hasta la muerte por esclarecer los hechos.

Los medios audiovisuales dejan espacio a las mujeres, puesto que hay en esta etapa tres mujeres con papel principal en ellos: en radio en $A l$ servicio de la mujer española y en Los últimos golpes del Torete III y en televisión en Las huellas del lince.

En los años 90 los héroes prosiguen con los dramas en los 8 largometrajes y sigue el dominio de la prensa. Para finalizar, en el siglo XXI de nuevo el drama impera y por primera vez la mujer supera al hombre con 8 casos y 7 hombres en las películas analizadas. Continúa la tendencia de las periodistas en el medio televisivo y de los 4 casos de este medio, 3 están protagonizados por mujeres. Películas como Yoyes, Soldados de Salamina o GAL se encuentran en esta etapa. 


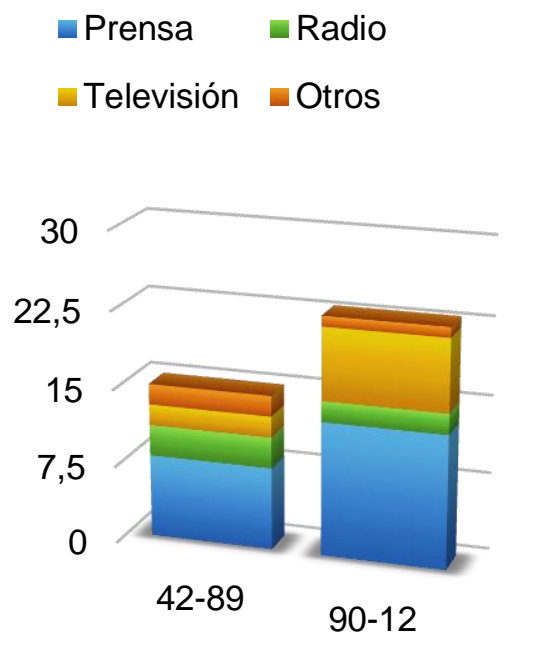

Gráfico 2. Héroes por medio de comunicación

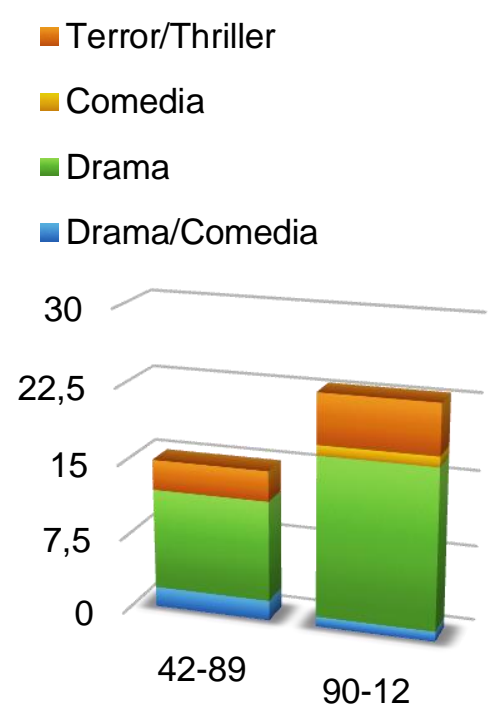

Gráfico 3. Héroes por géneros

$$
\begin{aligned}
& \text { - Hombres y mujeres } \\
& \text { Mujeres } \\
& \text { - Hombres }
\end{aligned}
$$

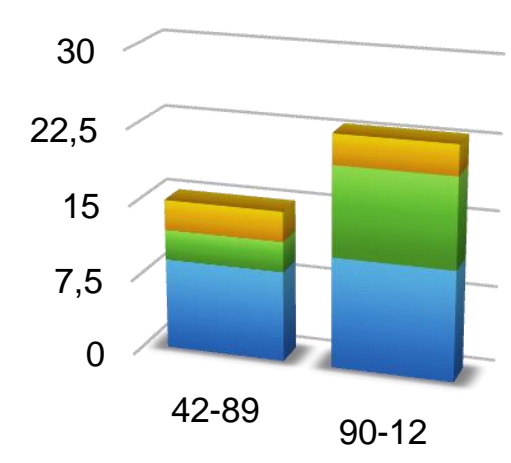

Gráfico 4. Héroes por sexos

\subsection{Periodista villano en situaciones realistas}

Los villanos de la prensa también registran una transformación a lo largo de las siete décadas de análisis. De las 66 películas que se consideran realistas por presentar periodistas que se pueden hallar en las redacciones de periódico, radio o televisión, en 30 casos son villanos.

En las décadas de los 40 y 50 se recogen 5 historias con villanos y se abre paso el género de la comedia con El ladrón de guante blanco. Los otros 4 casos restantes presentan drama con momentos cómicos. El medio de comunicación que prevale es la prensa aunque se encuentra el primer caso de radio, Esa voz es una mina. En estos 20 años con villanos no aparecen mujeres.

Como en las décadas anteriores, en los años 60 continúa el predomino de dramacomedia con 4 películas mientras que 2 de ellas son solo comedia, una de ellas la reconocida Plácido. De las 3 mujeres villanas que aparecen en esta etapa, 2 se muestran en los 2 casos televisivos que se concentran en esta etapa, Cuando tú no estás y Las cuatro bodas de Marisol. Hay una mujer más en revista especializada, Relaciones casi públicas. Los 3 largometrajes con periodistas masculinos se reparten dos periódicos y el único caso de radio.

Con los 80 llegan los primeros auténticos paparazi con Corazón de papel, con Tomás Pineda y Nicolás Martín y sus trabajos sucios para una agencia, y en La vida alegre el periodista de una revista roba un informe médico de un consejero de Sanidad para 
conseguir así más lectores. La prensa es el escenario elegido por esos paparazis del cine y continúa el tono de comedia para los primeros villanos, todos ellos hombres.

En los años 90, a los 3 dramas con toques de comedia se suma en esta etapa un género que aún no había aparecido entre los villanos, el thriller, con 2 películas. Hay dominio de la mujer, como en las 11 películas de los primeros 12 años del siglo XXI.

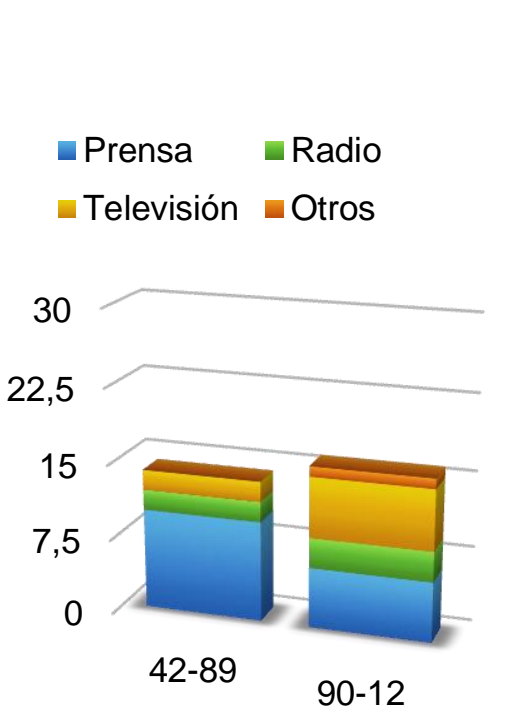

Gráfico 5. Villanos por medio de comunicación
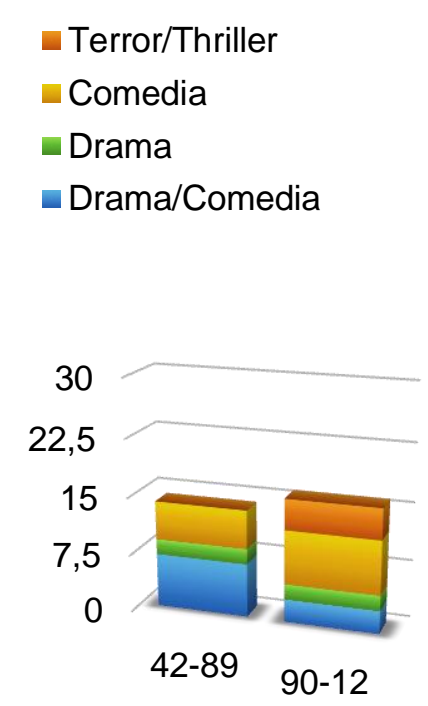

Gráfico 6. Villanos por géneros

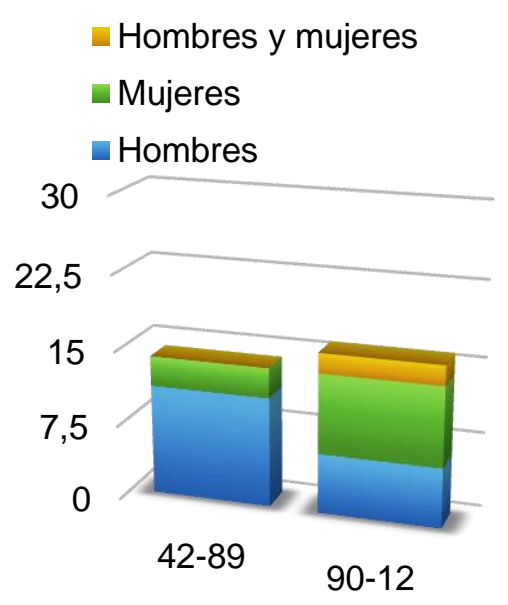

Gráfico 7. Villanos por sexos

\subsection{Periodista héroe en situaciones no realistas}

Los periodistas héroes que presenta el cine español en ocasiones surgen en situaciones no reales o al menos muy infrecuentes. En 32 películas aparecen este tipo de informadores y solo en 10 hay héroes. Son los menos frecuentes en la muestra de cine español seleccionada y en todos los casos están ligados al género de terror o misterio, siempre con tintes dramáticos.

El género fantástico, en el que suceden monstruos, fantasmas y otros seres del más allá o miembros de peligrosas sectas, se inmiscuye en la vida de los informadores, que actúan con valentía y compromiso. El terror tarda en llegar al cine español y más aún en confluir con un papel de periodista. La primera muestra aparece en 1980 con La invasión de los zombies atómicos (Umberto Lezi, 1980), en la que un periodista de televisión tiene que enfrentarse a los extraños muertos vivientes que en unas horas han inundado su ciudad.

En los años 90 la moda del terror deja tres títulos: uno de ellos con fantasmas y los otros dos abarcan el peligro de los grupos sociales como las sectas o juegos de rol. El primer caso es una mujer que trabaja en la radio, 99.9, y los dos segundos hombres en 
prensa: Los sin nombre y Nadie conoce a nadie. Por último, del año 2000 a 2012 surgen nuevas formas de terror y misterio con una periodista 'poseída', $R E C$, y otras dos con poderes extraños, Uno de los dos no puede estar equivocado y Bajo aguas tranquilas, los tres casos informadoras de televisión.

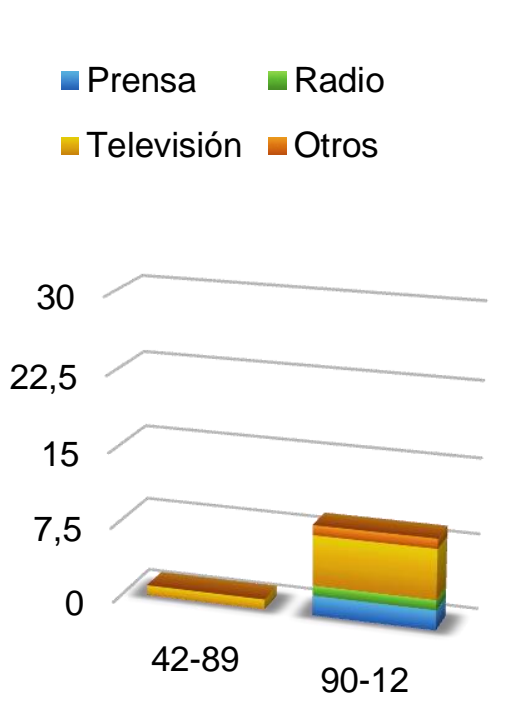

Gráfico 8. Héroes por medio de comunicación
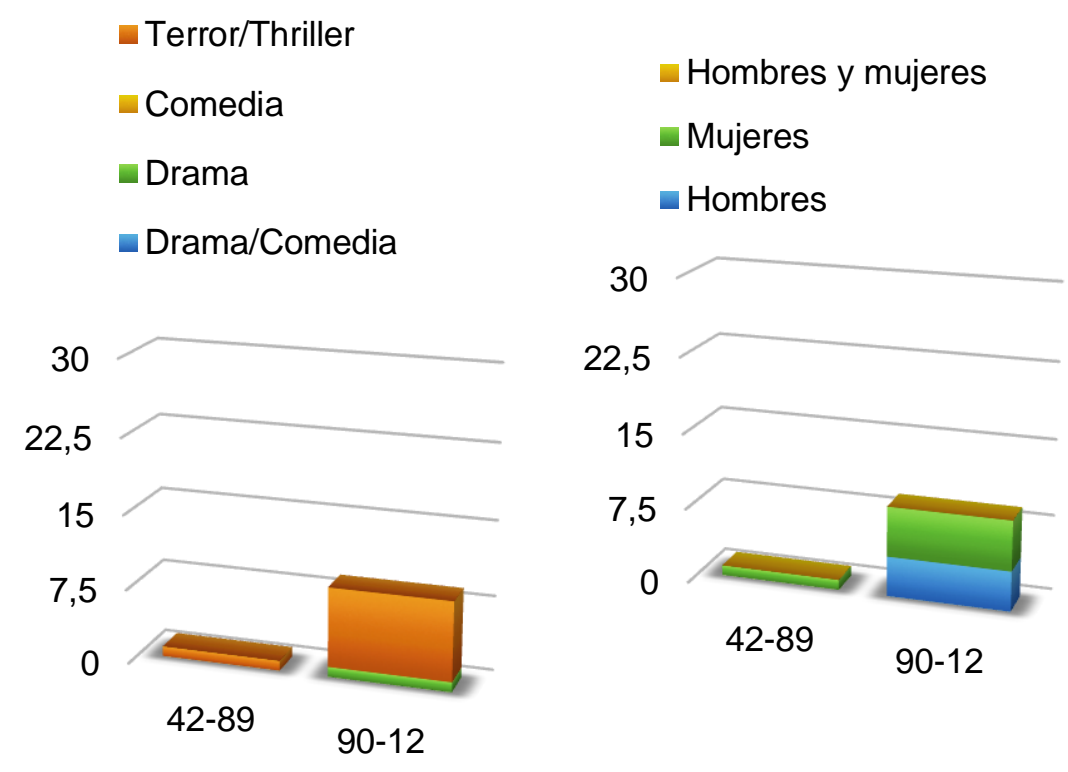
Gráfico 9. Héroes por géneros
Gráfico 10. Héroes por sexos

\subsection{Periodista villano en situaciones no realistas.}

Los periodistas villanos que plasma el cine español también surgen en situaciones que a primera vista no parecen realistas o al menos son muy poco frecuentes. Se presentan en 22 casos.

En los años 60 aparece el primer título con Una tal Dulcinea, una película en la que sátira y humor acompañan a un periodista "multitarea" que colabora en un diario y una televisión. Como con cuentagotas se presenta en los 80 el segundo caso, esta vez un hombre que trabaja en la radio y el humor se mezcla con el erotismo, La radio habla (Radio Speed).

En la década de los 90 se reafirma el humor entre los villanos con mentiras, noticias del corazón y televisión como telón de fondo. La comedia rige 8 títulos, en 2 comparte pantalla con el drama y en 2 solo hay drama. En los doce primeros años del nuevo siglo, cobra fuerza la unión televisión-mujer-maldad. La mujer villana se presenta en 6 ocasiones de las 8 que películas que cierran la muestra de malvados. Tacones lejanos, Kika, Dame algo o El grito en el cine son algunas de ellas. 

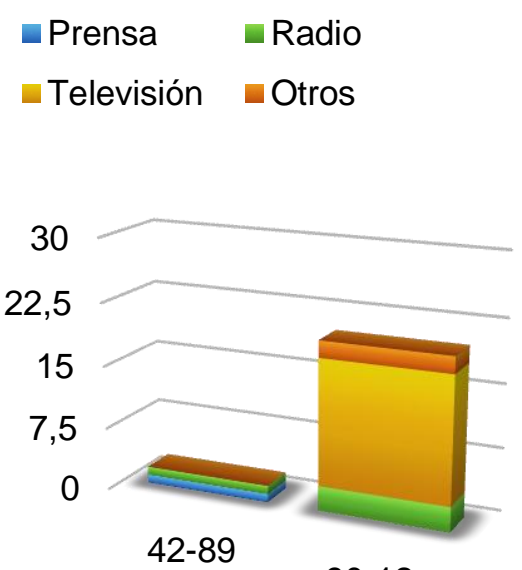

$90-12$

\section{- Terror/Thriller \\ - Comedia \\ - Drama}

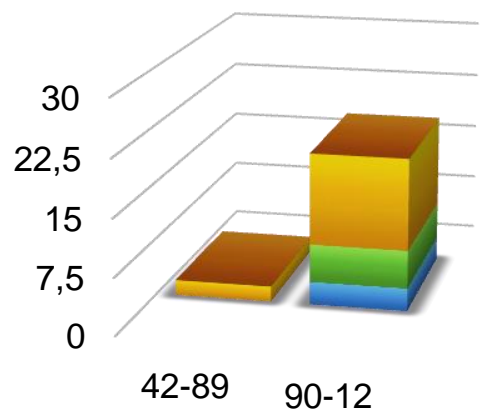

- Hombres y mujeres

- Mujeres

- Hombres

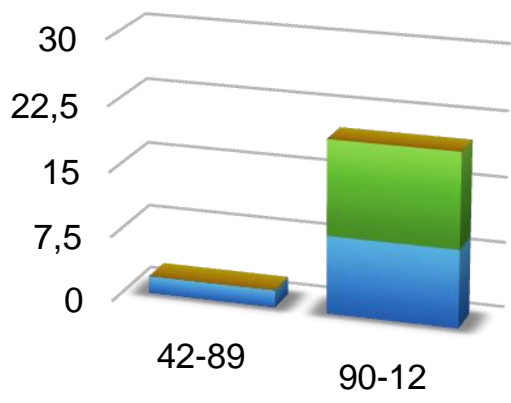

Gráfico 11. Villanos por medio de comunicación
Gráfico 12. Villanos por

géneros

Gráfico 13. Villanos por

sexos

\section{CONCLUSIÓN Y DISCUSIONES}

Con el estudio de las 135 películas españolas a lo largo de los 70 años (1942-2012) realizado durante esta investigación, se han ido cumpliendo los objetivos dirigidos a estructurar una morfología de los profesionales de los medios de comunicación que permita identificar el perfil del periodista y su evolución desde 1942 hasta 2012. Las 21 funciones extraídas, que siguen el modelo expuesto por Vladimir Propp en Morfología del cuento (1928), aparecen en las películas en las que el periodismo es el hilo conductor o detonante de sus historias, funciones que aportan una original semiosis.

En el $72 \%$ de las 135 películas con periodistas, se muestran claros ejemplos de héroes o villanos de la profesión. El cine refleja buenos y malos del periodismo en una cifra similar en ambos bandos, 48 en el primer caso y 52 en el segundo.

Aunque la cifra de películas con villanos supera ligeramente a la de los héroes, en un análisis más pormenorizado de la presencia de malos y buenos se detecta que predominan los héroes cuando las películas presentan dramas en situaciones realistas y los villanos cuando se producen las situaciones no realistas, es decir cuando se realiza comedia con exceso de sátira. Por tanto, se verifica H1: las funciones del periodista como profesional en su puesto de trabajo reflejan la dicotomía entre héroes enmarcados en el género drama y villanos en el género comedia. 


\section{San José de la Rosa, C \\ Estructuralismo de Propp en los periodistas héroes y villanos del cine español}

Con toda la información recabada sobre el ejercicio de la profesión, se puede afinar aún más la presencia de héroes y villanos en el cine y relacionar esa bondad o maldad no solo con características como la modalidad cinematográfica sino también con factores como el medio de comunicación y el género masculino o femenino. A continuación se exponen las conclusiones en los cuatro epígrafes analizados en los resultados:

- El drama, la prensa escrita y los varones dominan entre los héroes en situaciones realistas hasta la Transición. A partir de 1975, en el apartado de héroes surge con fuerza el periodismo de opinión y también con ímpetu aparece un medio de comunicación, la radio. Hombres y mujeres empiezan a compartir protagonismo en la gran pantalla, pero mientras que ellos siguen su dominio en la prensa escrita con informaciones comprometidas e incluso hay dos hombres que llegan a morir por descubrir la verdad, las mujeres ocupan el medio televisivo y temáticas más banales.

- En los villanos en situaciones realistas, llama la atención la aparición de periodistas femeninas antes, ya en los 60, y líder del nuevo medio, la televisión. Ese tándem mujer e imagen frívola en la pantalla va en aumento a lo largo del periodo estudiado hasta llegar a la actualidad.

- En el caso de héroes en situaciones no realistas, además de dramas hay destacada presencia de thriller y terror, el reparto de hombres y mujeres es equitativo y de nuevo ellas dominan en televisión.

- Por último, el número de villanos en situaciones no realistas es el doble que el de los héroes y además del miedo se opta también por la sátira con comedias que rozan el absurdo. De nuevo se produce la unión televisión, mujer y maldad, sobre todo en el siglo XXI.

Con los resultados resultados de los cuatro grupos también se verifica H2: la prensa escrita es el medio de comunicación dominante. Los periódicos son el medio de comunicación más abundante y gozan de más prestigio en todas las etapas estudiadas. La radio se mantiene con una discreta presencia y cuenta con películas emblemáticas, sobre todo en el camino a la democracia. A partir de 1990 se observa un fuerte incremento de la televisión con la aparición de las cadenas privadas, que desde este momento y hasta 2012 consigue superar mínimamente a la prensa escrita. No hay rastro de los nuevos géneros digitales y de la revolución que ha supuesto internet en las formas de comunicación.

También a partir de los datos de los cuatro grupos de películas se verifica $\mathrm{H} 3$, la discriminación profesional favorece a los varones. En las primeras décadas de estudio, el escaso número de casos femeninos no deja establecer comparaciones entre hombres y mujeres y su representación. Se observa que las dos primeras comunicadoras surgen en los años 60 y se encargan de anticipar el papel que ocupará la mujer periodista: asuntos turbios y frívolos. Tanto una como otra pretenden convertir a dos cantantes en estrellas nacionales a través de mentiras y manipulaciones. 
Ya en los 90, la presencia contundente de la mujer, con algo más de la mitad de los casos de periodistas, permite detectar la preferencia de los directores de cine por asignar a las féminas papeles de periodistas sin ética ni principios. Mujeres y televisión configuran esa unión perjudicial y el $47 \%$ de las periodistas en los últimos 22 años son presentadoras en la pequeña pantalla. Son 29 y solo 6 realizan televisión comprometida mientras que las otras 23 se dedican al entretenimiento o temas banales.

En el mismo periodo, El País o Diario 16 aparecen una y otra vez para ubicar a los reputados periodistas. Hasta el 51\% de los hombres en las películas de 1990 a 2012, un total de 25 , trabajan en prensa escrita bien valorada.

En definitiva, 135 películas con héroes y villanos de la prensa que muestran la evolución de los medios de comunicación con perjuicio para la mujer y para el medio televisivo, una tendencia que habrá que vigilar con el análisis de las películas desde 2012 a 2021. Podría cambiar gracias a las historias con valientes mujeres como las de las periodistas televisivas Virginia Vallejo (Penélope Cruz) en Loving Pablo (Fernando León de Aranoa, 2017) o Amaia Marín (Bárbara Lennie) en la aclamada El Reino (Rodrigo Sorogoyen, 2018).

\section{REFERENCIAS}

Aumont, J., Bergara, A., Marie, M. y Vernet, M. (1985). Estética del cine: espacio fílmico, montaje, narración, lenguaje. Barcelona. Paidós Ibérica.

Aumont, J. y Marie, M. (1988). Análisis del filme. Barcelona. Paidós Comunicación.

Barris, A. (1976). Stop the presses! The newspaperman in American Films. Cranbury, New Jersey. A. S. Barnes and Co.

Barthes, R., Greimas, J., Eco, U., Gritti, J., Morin, V., Metz, C., Genette, G., Todorov, T., \& Bremond, C. (s. f.). Introducción al análisis estructural de los relatos. En Análisis estructural del relato. Coyoacán

Ehrlich, M. C. (2006). Journalism in the movies. Illinois (EEUU). University of Illinois Press.

Ehrlich, M. C. y Saltzman, J. (2015). Heroes and Scoundrels: The Image of the Journalist in Popular Culture. Chicago. University of Illinois Press.

Good, H. (1989). Outcasts: The image of the journalists in contemporary filme. London. The Scarecrow Press.

Good, H. (2008). Journalism ethics goes to the movies. Maryland (EEUU). Rowman and Littlefield. 
McNair, B. (2010). Journalists in filme. Heroes and Villains. Edinburgh. Edinburgh University Press Ltd.

Mera Fernández, M. (2008). Periodistas de película. La imagen de la profesión periodística a través del cine. Revista Estudios sobre el mensaje periodístico, 14, 505525.

Miguel Borrás, M. (2014). El cine como instrumento ideológico: la Seminci (19561975). Delgado Idarreta, J.M.; Pérez Serrano, J. y Viguera Ruiz, R. (eds.). Iglesia y Estado en la sociedad actual. Política, cine y religión. Logroño. Instituto de Estudios Riojanos.

Miguel Borrás, M. (2008). La poética del cine. Siete miradas, una misma luz. Teoría y análisis cinematográfico. J. Bermejo y M. Canga (coords.) Valladolid. Universidad de Valladolid.

Monterde, J. E., Selva, M. y Solà, A. (2001). La representación cinematográfica de la historia. Madrid. Akal.

Orellana, J. (2004). Como en un espejo. Drama humano y sentido religioso en el cine contemporáneo. Madrid. Ediciones Encuentro.

Pastor-Alonso, M.-Á. (2010). I want to be a journalist: Looking for motivation in journalism. Comunicar, 17(34), 191-200. https:/ / doi.org/10.3916/C34-2010-03-19

Propp, V. (1971). Morfología del cuento. Madrid. Editorial Fundamentos.

Propp, V. (2011). Morfología del cuento. Madrid. Akal.

Rodríguez-Merchán, E. (2007). Cinema teaching in Spain. A historical perspective and a contemporary view. Comunicar, 15(29), 13-20. https://doi.org/10.3916/C292007-01

San José-De la Rosa, C. (2017). El perfil del periodista en el cine español (1942-2012). Tesis doctoral presentada en la Universidad de Valladolid. Sin publicar.

San José-De la Rosa, C., Miguel-Borrás, M. y Gil-Torres, A. (2019). Representación del periodista en el cine español desde 1990 hasta 2010. Doxa Comunicación, 29, 139159. https://doi.org/10.31921/doxacom.n29a7

San José-De la Rosa, C.; Miguel-Borrás, M. y Gil-Torres, A. (2020). Periodistas en el cine español: héroes comprometidos con la verdad. Estudios sobre el Mensaje Periodístico, 26(1), 317-326. https://doi.org/10.5209/esmp.67310

Tello, L. (2011). La imagen y la ética del periodista en el cine español (1896-2010). Tesis doctoral presentada en la Universidad Complutense de Madrid. Sin publicar. 
Tello, L.(2016). Hablemos de cine. 20 cineastas conversan sobre el cuarto poder. Zaragoza. Prensas Universitarias de Zaragoza.

William-Evans, P. (2007). Film teaching in the British Educational System. Comunicar, 15(29), 27-29. https:// doi.org/10.3916/C29-2007-03

\section{AUTORA}

\section{Cristina San José de la Rosa:}

Doctora profesora en el Grado de Periodismo de la Universidad de Valladolid y en el Máster de Cine de la Universidad de Valladolid. Licenciada en Ciencias de la Información (Universidad Pontificia de Salamanca) y en Filología Hispánica (Universidad de Salamanca). Trayectoria profesional durante 22 años en El Norte de Castilla de Valladolid, Antena 3 Castilla y León y El Mundo de Castilla y León. En 2006 obtuvo el Premio de Periodismo Provincia de Valladolid. Tesis doctoral sobre periodismo en el cine español en 2017 y líneas de investigación relacionadas con la presencia del periodista en el cine y el periodismo cinematográfico. Desde 2013 es profesora de Redacción Períodóstica, Géneros Periodísticos Interpretativos, Periodismo Cultural y Teorías de la Información en el Grado de Periodismo de la Universidad de Valladolid. Colaboraciones periodísticas en las secciones de Cultura de El Mundo de Castilla y León, en el periódico digital Tribuna de Valladolid y en Radio Televisión Castilla y León.

cristina.sanjose@uva.es

Orcid ID: https://orcid.org/0000-0001-6891-3170 